\title{
Orientation dependence of the pseudo-Hall effect in p-type 3C-SiC four-terminal devices under mechanical stress
}

\author{
Hoang-Phuong Phan, ${ }^{* a}$ Afzaal Qamar, ${ }^{a}$ Dzung Viet Dao, ${ }^{a, b}$ Toan Dinh, ${ }^{a}$ Li Wang, \\ Jisheng Han, ${ }^{a}$ Philip Tanner, ${ }^{a}$ Sima Dimitrijev, ${ }^{a, b}$ and Nam-Trung Nguyen ${ }^{a}$
}

Received * * 2015

DOI: 10.1039/b000000x

This paper presents for the first time the orientation dependence of the pseudo-Hall effect in p-type $3 \mathrm{C}$-SiC fourterminal devices under mechanical stress. Experimental results indicate that the offset voltage of p-type $3 \mathrm{C}-\mathrm{SiC}$ four-terminal devices significantly depends on the directions of the applied current and stress. We also calculated the piezoresistive coefficients $\pi_{61}, \pi_{62}$, and $\pi_{66}$, showing that $\pi_{66}$ with its maximum value of approximately $16.7 \times 10^{-11} \mathbf{P a}^{-1}$ plays a more dominant role than $\pi_{61}$ and $\pi_{62}$. The magnitude of the offset voltage in arbitrary orientation under stress was estimated based on these coefficients. The finding in this study plays an important role in the optimization of Microelectromechanical Systems (MEMS) mechanical sensors utilizing the pseudoHall effect in p-type 3C-SiC.

Silicon Carbide (SiC), with its large energy band gap (2.3-3.4 $\mathrm{eV}$ ) and superior mechanical properties, is an excellent candidate for electrical devices operating in harsh environments ${ }^{1-5}$. Recent studies have paid significant attention to the characterization of the strain effect on $\mathrm{SiC}$ for sensing applications under hostile conditions ${ }^{6-8}$. Many groups have reported the piezoresistive effect in various poly types of $\mathrm{SiC}$ such as $3 \mathrm{C}$ $\mathrm{SiC}, 4 \mathrm{H}-\mathrm{SiC}$, and $6 \mathrm{H}-\mathrm{SiC}$. Large gauge factors of approximately 30 were reported in both p-type and n-type single crystalline $\mathrm{SiC}$, indicating that $\mathrm{SiC}$ has high potential for mechanical sensing applications ${ }^{9-14}$. Among more than 200 poly types of crystalline $\mathrm{SiC}$, cubic crystalline silicon carbide (3C-SiC) is preferable for Microelectromechanical Systems (MEMS) transducers. The main advantage of 3C-SiC over other poly types is the capability of growth on a silicon ( $\mathrm{Si}$ ) substrate, which reduces the cost of wafers and is more compatible with the conventional MEMS process ${ }^{2,12-15}$. Most previous studies on the piezoresistive effect of $\mathrm{SiC}$ utilized the conventional two-terminal resistors requiring a Wheatstone bridge for voltage read-out ${ }^{15,16}$, which faces several drawbacks. For instance, the four resistors in the bridge must be closely identical to obtain a zero offset ${ }^{17-19}$. In addition, the resistors in

\footnotetext{
*Emails of corresponding authors: hoangphuong.phan@ griffithuni.edu.au Hoang-Phuong Phan and Afzaal Qamar share equal contribution to this article.

${ }^{a}$ Queensland Micro-Nanotechnology Centre, Griffith University, Queensland, Australia.

${ }^{b}$ School of Engineering, Griffith University, Queensland, Australia.
}

the Wheatstone bridge must have almost the same temperature coefficient to avoid the offset drift due to the change of temperature ${ }^{20}$. Compared to two-terminal resistors, four-terminal devices have proved to be more thermally stable and suitable for the miniaturization of sensors, since they do not require any external Wheatstone bridge ${ }^{17-21}$. Under a mechanical shear strain, a voltage is generated across two terminals of four-terminal devices due to the distortion of potential distribution, and this phenomenon has been named as the pseudoHall effect ${ }^{22,23}$. To date, there have been various studies on the pseudo-Hall effect in silicon material, and in fact this effect in Si has been utilized in commercial strain sensors for more than two decades ${ }^{23,24}$. However, the work on the pseudo-Hall effect in $\mathrm{SiC}$ has been rarely reported ${ }^{25}$. As the pseudo-Hall effect in semiconductors depends on the crystallography orientation, it is important to investigate which orientation offers the most significant effect in $3 \mathrm{C}-\mathrm{SiC}$ four-terminal devices.

In this paper, we report on the orientation dependence of the pseudo-Hall effect in p-type 3C-SiC thin films. The piezoresistive coefficients, $\pi_{61}, \pi_{62}$, and $\pi_{66}$, which define the magnitude of the pseudo-Hall effect were also investigated. The insight gained in this study is vital to the development of MEMS mechanical sensors using the pseudo-Hall effect in 3C-SiC.

The 3C-SiC thin films were grown on (100) Si substrate using low pressure chemical vapor deposition (LPCVD) ${ }^{26}$ at a low temperature of $1000{ }^{\circ} \mathrm{C}$. The alternating supply epitaxy approach was used to achieve single crystalline $\mathrm{SiC}$ film deposition with silane $\left(\mathrm{SiH}_{4}\right)$ and propylene $\left(\mathrm{C}_{3} \mathrm{H}_{6}\right)$ as precursors. Aluminum was employed as the dopant in the in situ doping process where trimethylaluminum $\left[\left(\mathrm{CH}_{3}\right)_{3} \mathrm{Al}\right.$, TMAl] was the precursor to form p-type $3 \mathrm{C}$-SiC. The properties of the grown single crystalline $3 \mathrm{C}$-SiC films were characterized and reported elsewhere ${ }^{26,27}$. The X-ray diffraction (XRD) measurement indicated that the $\mathrm{SiC}$ film is epitaxially grown on Si (100) substrate, Fig. 1 (a). Based on the X-ray photoelectron spectroscopy data, the atomic concentrations of carbon and silicon were found to be approximately $52.9 \%$ and 47.1 $\%$, respectively, while that of aluminum was below $1 \%$. The atomic force microscopy (AFM) image of a $380 \mathrm{~nm} \mathrm{3C-SiC}$ film shows that the roughness of a $5 \mu \mathrm{m} \times 5 \mu \mathrm{m}$ area was $20 \mathrm{~nm}$, Fig. 1 (b). The semiconductor type and carrier concentration of the films were then investigated using the hot probe method $^{28}$. The positive voltage at the hot probe indi- 
(a) XRD

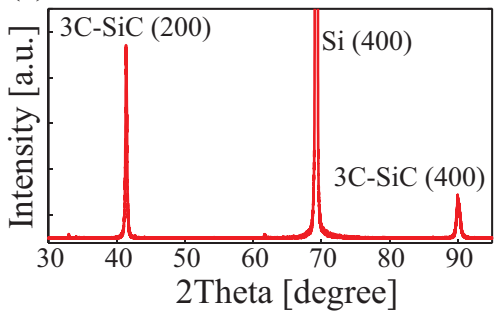

(b) AFM

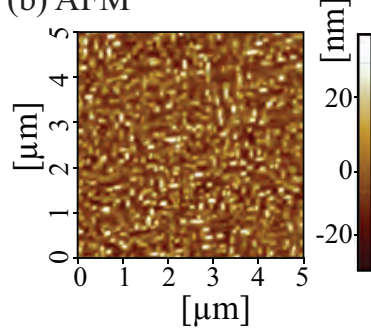

(c) Fabrication

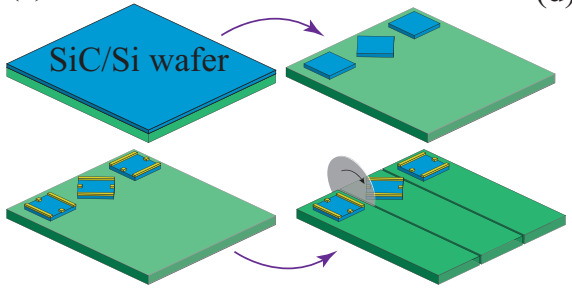

(d) Bending experiment

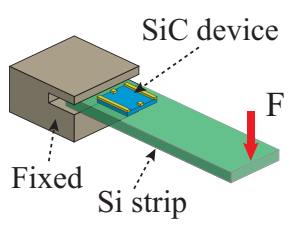

(e) Photograph of device

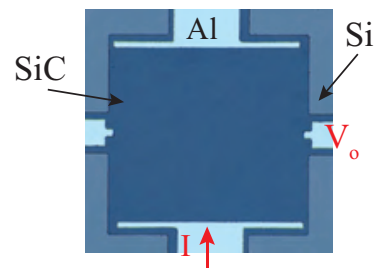

(f) Directions of current and stress

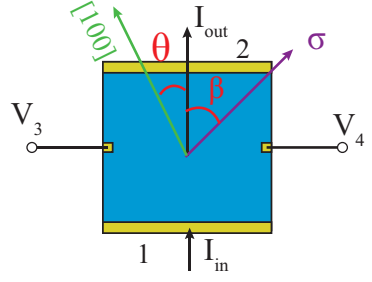

Fig. 1 (a) The XRD graph of the 3C-SiC film grown using the LPCVD process; (b) The AFM image of a $380 \mathrm{~nm} \mathrm{SiC} \mathrm{film;} \mathrm{(c)} \mathrm{The}$ fabrication process; (d) The bending experimental setup; (e) The photograph of a $\mathrm{SiC}$ four-terminal device with its dimensions of 500 $\mu \mathrm{m} \times 500 \mu \mathrm{m}$; (f) Schematic sketch of the directions of the current, voltage and applied stress.

cated the $3 \mathrm{C}$-SiC film was p-type semiconductor, and the carrier concentration was calculated to be in the range of $10^{18}$ $\mathrm{cm}^{-3}$. Figure 1 (c) shows the fabrication of the $3 \mathrm{C}-\mathrm{SiC}$ fourterminal device using a 2-masks-photolithography process. In the first step, $\mathrm{SiC}$ pattern was formed using Inductively Coupled Plasma (ICP) etching with an etch-rate of approximately $200 \mathrm{~nm} / \mathrm{min}^{27}$. Next, aluminum was deposited on the $\mathrm{SiC}$ film using sputtering, and aluminum electrodes were then patterned using wet etching. Finally, the $\mathrm{SiC} / \mathrm{Si}$ wafers were diced into smaller strips with dimensions of $9 \mathrm{~mm} \times 60 \mathrm{~mm}$ $\times 0.6 \mathrm{~mm}$ for the subsequent bending experiment, Fig. 1 (d). A photograph of the fabricated devices and a schematic sketch of the current and applied stress are shown in Fig. 1 (e)(f).

The linear current-voltage characteristics of the $\mathrm{SiC}$ fourterminal devices were measured using ${ }^{\mathrm{TM}} \mathrm{HP} 4145 \mathrm{~B}$, indicating a good Ohmic contact between the aluminum electrodes and the p-type $3 \mathrm{C}-\mathrm{SiC}$ films. The current leakage between the $3 \mathrm{C}-\mathrm{SiC}$ layer and the $\mathrm{Si}$ substrate was also investigated to ensure that Si substrate did not contribute to the measured results (See the supporting information). To characterize the pseudoHall effect in the four-terminal $\mathrm{SiC}$ devices, we applied stress to the $\mathrm{SiC}$ films using the bending beam method, while sup-

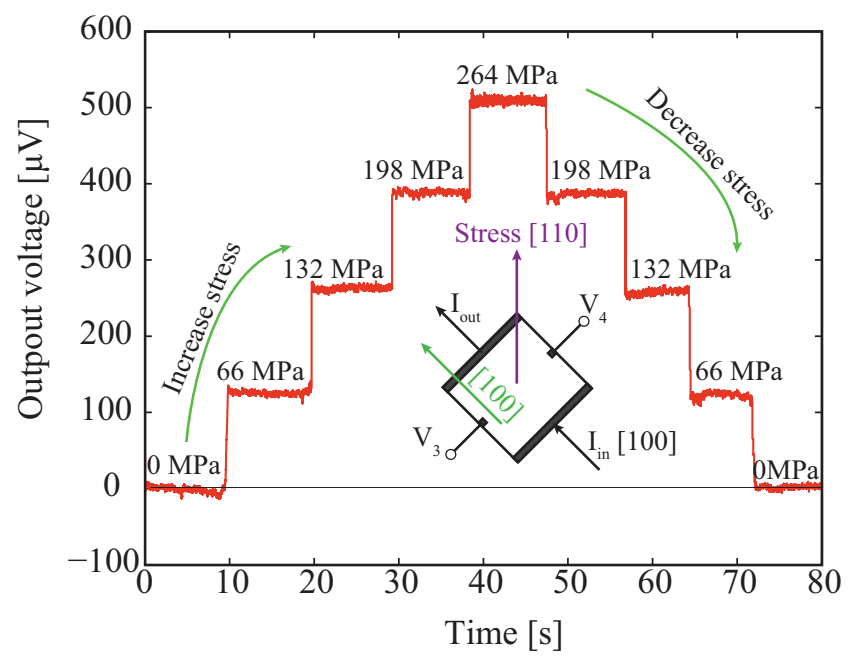

Fig. 2 The generated voltage across terminals 3 and 4 when supplying a constant current of $10 \mu \mathrm{A}$ through terminals 1 and 2 of a $\mathrm{SiC}$ four-terminal devices under several applied stresses from 0 to $264 \mathrm{MPa}$. Note that the orientation of the current is [100], while that of the applied stress is [110] in (100) plane.

plying a constant current through terminals 1 and 2 and monitoring the change of the generated voltage across terminal 3 and 4 of the SiC devices, Fig. 1 (d)(f). Since the thickness of the SiC films $(\sim 300 \mathrm{~nm})$ is less than $0.1 \%$ of that of the Si substrate $(600 \mu \mathrm{m})$, we assumed that the strain induced into the $\mathrm{SiC}$ films is approximately equal to that of the surface of the Si substrate: $\varepsilon=M t / 2 E_{S i} I$, where $M$ is the bending moment; $I$ is the moment of inertia; $E_{S i}$ and $t$ are the Young's modulus and the thickness of the Si layer, respectively. Accordingly, for an applied load varied from 0 to $2 \mathrm{~N}$ to the free end of the $\mathrm{Si}$ strips, the strain induced into the $\mathrm{SiC}$ layer was in a range of 0 to $900 \mathrm{ppm}$. The stress applied to the $\mathrm{SiC}$ layer $\left(\sigma_{S i C}\right)$ was then calculated using Hooke's law: $\sigma_{S i C}=E_{S i C} \varepsilon$, where $E_{S i C}$ is the Young's modulus of $3 \mathrm{C}-\mathrm{SiC}^{29}$.

We investigated the orientation dependence of the pseudoHall effect of p-type 3C-SiC thin films by characterizing $\mathrm{SiC}$

Table 1 List of SiC four-terminal samples

\begin{tabular}{ccccc}
\hline $\begin{array}{c}\text { Test } \\
\text { samples }\end{array}$ & $\begin{array}{c}\text { Orientation } \\
\text { of current }\end{array}$ & $\begin{array}{c}\text { Orientation } \\
\text { of uniaxial stress }\end{array}$ & $\theta$ & $\beta$ \\
\hline \hline A & {$[100]$} & {$[110]$} & $0^{\circ}$ & $45^{\circ}$ \\
B & {$[110]$} & {$[110]$} & $45^{\circ}$ & $0^{\circ}$ \\
C & {$[110]$} & {$[1 \overline{1} 0]$} & $45^{\circ}$ & $90^{\circ}$ \\
D & {$[110]$} & {$[100]$} & $45^{\circ}$ & $45^{\circ}$ \\
E & {$[100]$} & {$[100]$} & $0^{\circ}$ & $0^{\circ}$ \\
F & {$[100]$} & {$[010]$} & $0^{\circ}$ & $90^{\circ}$ \\
\hline
\end{tabular}

$\theta$ is the angle between the direction of the applied current and [100] orientation, and $\beta$ is the angle between the directions of the applied stress and the current in 3C-SiC (100) plane. 


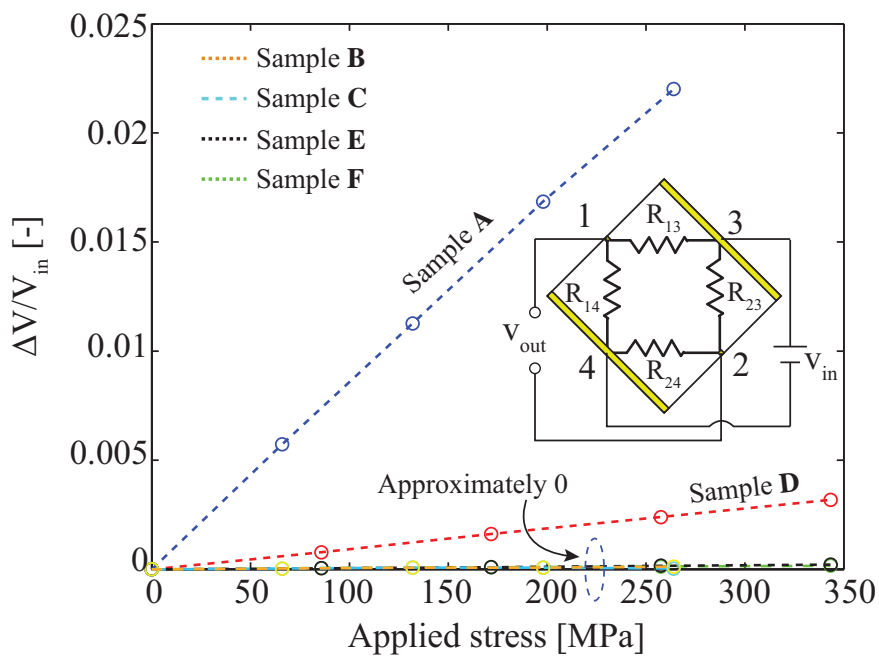

Fig. 3 The ratio of the generated voltage and applied voltage of the $\mathrm{SiC}$ four-terminal devices aligned in different orientation. Inset: The equivalent four resistors $R_{13}, R_{14}, R_{23}$, and $R_{24}$ of a $3 \mathrm{C}-\mathrm{SiC}$ four-terminal device ${ }^{32}$.

four-terminal devices aligned in different directions and being stressed in different orientations, as summarized in Table. I. Figure 2 shows that for sample A (the orientation of the current is [100], and the direction of the stress is [110]), an offset voltage was generated across terminals 3 and 4 when we applied a stress in [110] orientation. It is also clear that, the offset voltage increased when increasing the applied stress to $264 \mathrm{MPa}$. The offset voltage then decreased with decreasing the stress, and returned to 0 when the load was completely removed. The generated voltage of the $\mathrm{SiC}$ four-terminal resistors under stress was measured for several testing cycles, showing a good reproducibility without any significant drift voltage (See supplementary information). This indicates the feasibility of using the pseudo-Hall effect in p-type 3C-SiC four-terminal devices for MEMS strain/stress sensors.

The same phenomenon was also observed in other samples (B, C, D, E, F), but with different order of magnitude. Figure 3 shows the ratio of the output voltage to the input voltages of different samples A, B, C, D, E and F, indicating a linear relationship between the output offset voltage $\left(V_{\text {out }} / V_{\text {in }}\right)$ and applied stress $(\sigma)$. These results also show that the magnitude of the generated offset voltage in 3C-SiC four-terminal devices varies with the directions of the current and the applied stress, indicating the orientation dependence of the pseudoHall effect in p-type $3 \mathrm{C}$-SiC thin film. As 3C-SiC has the cubic crystalline structure like $\mathrm{Si}$, we qualitatively and quantitatively explained the pseudo-Hall effect and its orientation dependence in p-type 3C-SiC, using the model proposed by Kanda et al. for the case of $\mathrm{Si}^{17,30}$. Figure 3 (Inset) shows an equivalent circuit of the four-terminal device, which consists of four resistors $R_{13}, R_{14}, R_{23}$, and $R_{24}$. In the strainfree state, these four resistors are considered to be identical

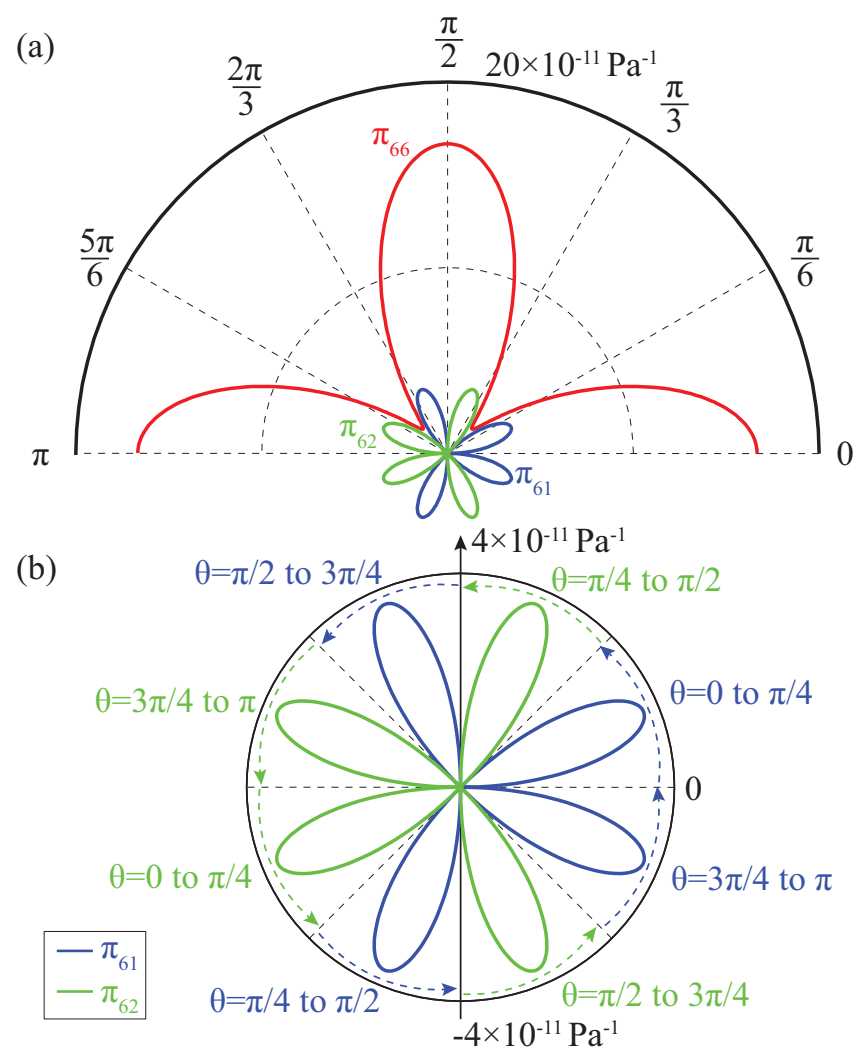

Fig. 4 (a) The coefficients $\pi_{61}, \pi_{62}$, and $\pi_{66}$ of SiC four-terminal devices when $\theta$ varies from 0 to $\pi$; (b) $\pi_{61}$ and $\pi_{62}$ are plotted in a different scale.

$\left(R_{13}=R_{14}=R_{23}=R_{24}\right)$. Under a uniaxial mechanical strain as shown in Fig. $1(\mathrm{~d})$, these four resistors are stressed, and their resistances change. As $R_{13}, R_{14}, R_{23}$, and $R_{24}$ are aligned in different orientations, these resistance changes are different, leading to an unbalance of the bridge circuit, which results in a non-zero voltage across terminals 3 and 4 . The ratio of the generated voltage $\left(V_{\text {out }}\right)$ across terminals 3 and 4 to the applied voltage across terminals 1 and $2\left(V_{i n}\right)$ due to stress can be quantified by the coefficients $\pi_{61}, \pi_{62}$, and $\pi_{66}$, as expressed in the following equation (see the supplementary information) ${ }^{33,34}$ :

$$
V_{\text {out }}=V_{\text {in }}\left(\pi_{61} \sigma_{1}+\pi_{62} \sigma_{2}+\pi_{66} \sigma_{6}\right)
$$

where $\sigma_{1}$ and $\sigma_{2}$ are normal stresses parallel and perpendicular to the current, and $\sigma_{6}$ is the in-plane shear stress which are calculated from the applied uniaxial stress $\sigma$, using Mohr's circle (see the supplementary information):

$$
\left\{\begin{array}{l}
\sigma_{1}=\sigma \cos ^{2} \beta \\
\sigma_{2}=\sigma \sin ^{2} \beta \\
\sigma_{6}=-\frac{\sin 2 \beta}{2} \sigma
\end{array}\right.
$$

here, $\beta$ is the angle between the applied uniaxial stress $(\sigma)$ and the current. The piezoresistive coefficients are defined in the 


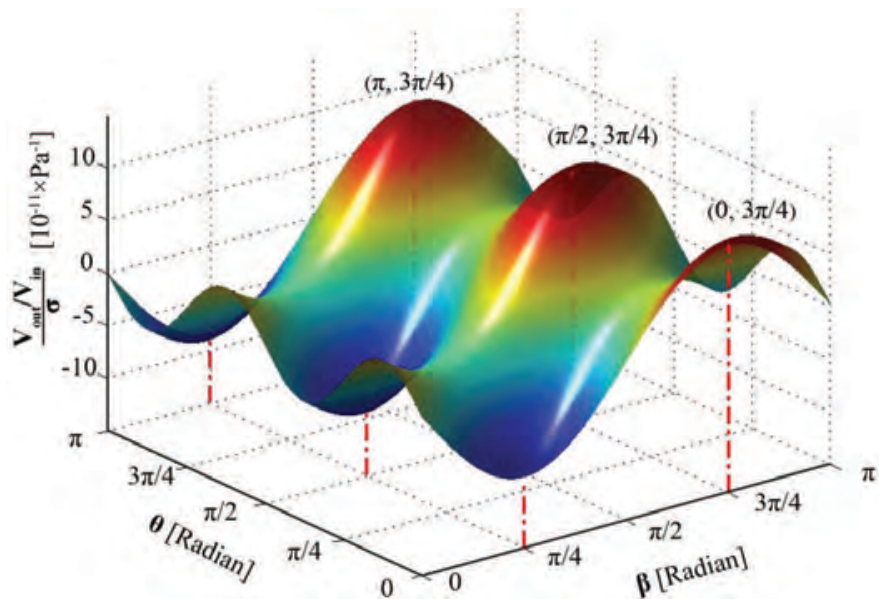

Fig. 5 The ratio of the generated voltage across terminals 3 and 4 to the input voltage across terminals 1 and 2 per unit uniaxial stress $\sigma$.

following equation ${ }^{30,31}$ (see the supplementary information):

$$
\left\{\begin{array}{l}
\pi_{61}=-\frac{1}{4} a \sin 4 \theta \\
\pi_{62}=\frac{1}{4} a \sin 4 \theta \\
\pi_{66}=b+a \sin ^{2} 2 \theta
\end{array}\right.
$$

where $\theta$ is the angle between the direction of the applied current and [100] orientation in (100) plane, and $a=\pi_{11}-\pi_{12}-$ $\pi_{44}$ and $b=\pi_{44}$ are constant parameters. Thus, by measuring the change of the generated voltage in samples A and D, we can determine parameters $a$ and $b$, and consequently, the piezoresistive coefficients $\pi_{61}, \pi_{62}$ and $\pi_{66}$. From the experimental results shown in Fig. 3, $a$ and $b$ were calculated to be $-14.8 \times 10^{-11} \mathrm{~Pa}^{-1}$ and $16.7 \times 10^{-11} \mathrm{~Pa}^{-1}$, respectively. As a result, substituting $a$ and $b$ into Eq. 3, the magnitude of the coefficients in any arbitrary orientation on (100) plane can be estimated, Fig. 4. These results show that for the p-type $3 \mathrm{C}$-SiC four-terminal devices, the coefficient $\pi_{66}$ plays a more dominant role than $\pi_{61}$ and $\pi_{62}$. The experimental data obtained in samples B, C, E, F was also in good agreement with the theoretical calculation that the output offset voltage was approximately 0 , since the coefficients $\pi_{61}$ and $\pi_{62}$ are 0 when $\theta$ is equal to 0 or $\pi / 4$ radian.

Based on the coefficients, we estimated the magnitude of the output offset voltage of 3C-SiC four-terminal devices under stress for MEMS mechanical sensors. Substituting Eq. 2 into Eq. 1, and using the calculated piezoresistive coefficients shown in Fig. 4, it is possible to theoretically estimate the magnitude of the pseudo-Hall effect of 3C-SiC four-terminal devices aligned in arbitrary orientations. Figure 5 shows the ratio of the generated voltage to the applied voltage per unit applied stress $\left(\left[V_{\text {out }} / V_{\text {in }}\right] / \sigma\right)$. It is obvious that, the magnitude of the effect in p-type 3C-SiC varies with orientations of current and stress. Accordingly, in (100) plane, the absolute offset voltage $\left(\left|V_{\text {out }}\right|\right)$ gains its maximum value when $(\theta, \beta)=(m \pi / 2,(2 n+1) \pi / 4)$, where $m$ and $n$ are integers. Therefore, when designing MEMS mechanical sensors using the pseudo-Hall effect in p-type 3C-SiC four-terminal resistor, these orientations should be chosen to enhance the sensitivity of sensors. On the other hand, for the Hall-devices where a constant offset voltage under external stress is desired, the orientations of $(\theta, \beta)=(m \pi / 4, n \pi / 2)$ should be selected to minimize the pseudo-Hall effect (here $m$ and $n$ are integers).

In conclusion, we reported on the pseudo-Hall effect in p-type 3C-SiC four-terminal devices, the orientation dependence, and piezoresistive coefficients $\pi_{61}, \pi_{62}$, and $\pi_{66}$. The maximum value of $\pi_{66}$ was found to be $16.7 \times 10^{-11} \mathrm{~Pa}^{-1}$, while that of $\pi_{61}$ and $\pi_{62}$ was $3.7 \times 10^{-11} \mathrm{~Pa}^{-1}$, implying that $\pi_{66}$ plays a more dominant role than the other coefficients. Based on these piezoresistive coefficients, it is possible to estimate the magnitude of the pseudo-Hall effect in any arbitrary orientation. Furthermore, the large output voltage at a certain applied current and stress along with a direct/simple signal-readout method indicate that pseudo-Hall effect in p-type 3C-SiC is a good candidate for MEMS applications. These findings in this study play an important role in designing/optimizing MEMS mechanical sensors as well as Hall devices utilizing p-type 3C-SiC material.

This work was performed in part at the Queensland node of the Australian National Fabrication Facility, a company established under the National Collaborative Research Infrastructure Strategy to provide nano and micro-fabrication facilities for Australia's researchers. This work has been partially supported by the Griffith University's New Researcher Grants.

\section{References}

1 P. M. Sarro, Sens. Actuators. A, 2000, 82(1-3), 210.

2 M. Mehregany, C. A. Zorman, N. Rajan, and C. H. Wu, Proc. IEEE, 1998, 86(8), 1594.

3 X. Zhang, Y. Chen, W. Liu, W. Xue, J. Li, and Z. Xie, J. Mater. Chem. C, 2013, 1, 6479-6486.

4 (a) S. Chen, P. Ying, L. Wang, F. Gao, G. Wei, J. Zheng, Z. Xie, and W. Yang, RSC Adv., 2014, 4(16), 8376-8382; (b) S. Chen, P. Ying, L. Wang, G. Wei, J. Zheng, F. Gao, S. Su, and W. Yang, J. Mater. Chem. C, 2013, 1(31), 4779-4784.

5 (a) S. Chen, P. Ying, L. Wang, G. Wei, F. Gao, J. Zheng, M. Shang, Z. Yang, W. Yang, and T. Wu, NPG Asia Mater, 2015, 7(1), e157; (b) S. Chen, P. Ying, L. Wang, G. Wei, and W. Yang, Appl. Phys. Lett., 2014, 105(13), 133106.

6 A. Qamar, P. Tanner, D. V. Dao, H. P. Phan, T. Dinh, IEEE Electron Device Lett., 2014, 35(12),1293-1295.

7 J. Bi, G. Wei, L. Wang, F. Gao, J. Zheng, B. Tang, and W. Yang, J. Mater. Chem. C, 2013, 1, 4514.

8 F. Gao, J. Zheng, M. Wang, G. Wei, and W. Yang, Chem. Commun., 2011, 47, 11993. 
9 R. Shao, K. Zheng, Y. Zhang, Y. Li, Z. Zhang, and X. Han, Appl. Phys. Lett., 2012, 101, 233109.

10 T. Akiyama, D. Briand, and N. F. Rooij, J. Micromech. Microeng., 2012, 22, 085034.

11 R. S. Okojie, A. A. Ned, A. D. Kurtz, and W. N. Carr, IEEE Trans. Electron Devices, 1998, 45(4), 785.

12 H. P. Phan, P. Tanner, D. V. Dao, N. T. Nguyen, L. Wang, Y. Zhu, and S. Dimitrijev, IEEE Electron Device Lett., 2014, 35(3), 399.

13 H. P. Phan, D. V. Dao, P. Tanner, N. T. Nguyen, J. S. Han, S. Dimitrijev, G. Walker, L. Wang, and Y. Zhu, J Matter. Chem. C, 2014, 2, 7176-7179.

14 J. S. Shor, D. Goldstein, and A. D. Kurtz, IEEE Trans. Electron Devices, 1993, 40(6), 1093.

15 H. P. Phan, D. V. Dao, L. Wang, T. Dinh, N. T. Nguyen, A. Qamar, P. Tanner, S. Dimitrijev, and Y. Zhu, J. Mater. Chem. C, 2015, 3, 1172-1176.

16 C. H. Wu, C. A. Zorman, and M. Mehregany, IEEE Sensors J., 2006, 6(2), 316.

17 Y. Kanda, Jpn. J. Appl. Phys., 1987, 26(7R), 1031.

18 A. V. Gridchin, and V. A. Gridchin, Sensors and Actuators A: Physical, 1997, 58(3), 219-223.

19 A. Mian, J. C. Suhling and R.C. Jaeger, "The van der pauw stress sensor," IEEE Sensors J., 2006, 6(2), 340-356.

20 M. Doelle, D. Mager, P. Ruther, and O. Paul, Sensors and Actuators A: Physical, 2006, 127(2), 261-269.

21 R. C. Jaeger, M. Motalab, S. Hussain, and J. C. Suhling, J. Electronic Packaging, 2014, 136(4), 041014.
22 S. Yue, W. A. Moussa, and L. R. Williston, Appl. Phys. A, 2014, 116(2), 409-414.

23 J. C. Doll, and B. L. Pruitt, "Piezoresistor design and applications," Springer, 2013, ISBN 978-1-4614-8516-2.

24 J.J.E. Gragg, "Silicon pressure sensor," U.S. Patent 4,317,126, 23, 1982.

25 A. Qamar, H. P. Phan, D. V. Dao, P. Tanner, T. Dinh, L. Wang and S. Dimitrijev, IEEE Electron Device Lett., 2015, 36(7), 708-710.

26 L. Wang, S. Dimitrijev, P. Tanner, and J. Zou, Appl. Phys. Lett., 2009, 94, 181909.

27 Y. Wang, S. Li, J. Han, W. Wen, H. Wang, S. Dimitrijev, and S. Zhang, RSC Advances, 2014, 4(97), 54441-54446.

28 P. Tanner, L. Wang, S. Dimitrijev, J. Han, A. Iacopi, L. Hold, G. Walker, Science of Advanced Materials, 2014, 6(7), 1542-1547.

29 F. Iacopi, G. Walker, L. Wang, L. Malesys, S. Ma, B. V. Cunning, and A. Iacopi, Appl. Phys. Lett., 2013, 102, 011908.

30 Y. Kanda, IEEE Transactions on electron devices, 1982, 29(1), 64-70.

31 W. G. Pfann, and R. N. Thurston, J. Appl. Phys., 1961, 32(10), 2008-2019.

32 Y. Kanda, and M. Migitaka, Physica Status Solidi (a), 1976, 35(2), K115-118.

33 Y. Kanda, and K. Yamamura, Sensors and Actuators, 1989, 183, 247-257.

34 D. V. Dao, T. Toriyama, J. Wells, and S. Sugiyama, Sens. Mater., 2002, 15, 113-135. 